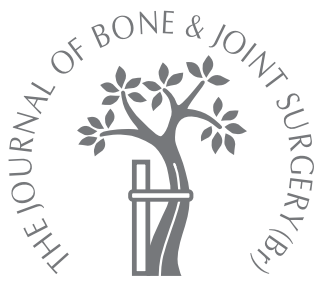

- CASE REPORT

\title{
Multifocal Proteus mirabilis osteomyelitis requiring bilateral amputation in an HIV- positive patient
}

\section{G. Petsatodis, P. D. Symeonidis, D. Karataglis, J. Pournaras}

From the "G. Papanikolaou" General Hospital, Thessaloniki, Greece

- G. Petsatodis, MD, PhD Associate Professor of Orthopaedics

I. P. Symeonidis, MD Orthopaedic Surgeon D. Karataglis, MD, $\mathrm{PhD}$ Orthopaedic Surgeon J. Pournaras, MD, PhD, Professor of Orthopaedics 1st Orthopaedic Department Aristotle University of Thessalonika, "G. Papanikolaou" Hospital, 57010 Exohi, Thessaloniki, Greece.

Correspondence should be sent to Dr P. D. Symeonidis; e-mail: p.symeonidis@gmail.com

(c)2007 British Editorial Society of Bone and Joint Surgery doi:10.1302/0301-620X.89B2 $18167 \$ 2.00$

$J$ Bone Joint Surg $[B r]$ 2007;89-B:249-51. Received 23 May 2006; Accepted after revision 12 September 2006

\begin{abstract}
We present a rare case of multifocal Proteus mirabilis osteomyelitis in an HIV-positive patient. Despite the patient's good immune status as assessed by her CD4 cell count and the aggressive treatment, she eventually underwent bilateral above-knee amputations to eradicate the infection. Multifocal Proteus mirabilis osteomyelitis can have an unpredictable clinical course with a severe outcome in HIV-positive patients.
\end{abstract}

Osteomyelitis caused by infection with Proteus mirabilis is rare. ${ }^{1-4}$ Its clinical course is unpredictable. To the best of our knowledge this is the first report of multifocal Proteus mirabilis osteomyelitis in an HIV carrier, as verified by repetitive cultures. We discuss its aggressive, but non-fatal clinical course.

\section{Case report}

A 43-year-old woman presented with a painful swelling in the anterior aspect of the proximal third of her right tibia. She had been diagnosed as being HIV-positive nine years earlier. She had a history of intravenous (IV) drug abuse and two suicide attempts, but to the best of our knowledge had not been using IV drugs for the previous five years. She had not experienced any clinical manifestations of AIDS and her CD 4 count as measured by flow cytometry had been steadily above 500 cells $/ \mathrm{mm}^{3}$ since the initial diagnosis. She had arbitrarily discontinued her antiretroviral medication four months before her first admission to hospital, and, despite medical advice, never resumed this. She also took regular antidepressant medication but no other drugs.

When seen she was walking with a limp, but was afebrile and normotensive. There was an abscess on the anterior aspect of the proximal right tibia. As an emergency procedure the abscess was incised and drained; it was found to communicate with the adjacent knee. Both the abscess cavity and knee were thoroughly debrided and irrigated. Cultures were obtained, which revealed infection with Proteus mirabilis. She received four intravenous injections of $500 \mathrm{mg}$ Imipenem (Mercu \& Co Ltd, Rayway, New Jersey) after the culturesensitivity results, according to the sensitivity results and remained in hospital. There was temporary relief of symptoms.

The erythrocyte sedimentation rate (ESR) and C-reactive protein (CRP) levels were elevated. Urine samples were negative for a subclinical Proteus mirabilis infection. Plain radiography and MRI of both knees revealed diffuse osteomyelitis of both tibiae and femora (Figs 1 and 2).

Ten days post-operatively the symptoms became more severe. She became febrile and complained of poorly localised pain throughout the right leg. On examination, there was redness and swelling over her right knee. A second debridement and drainage was performed. There was relief from pain and the fever subsided. Staged definite treatment was planned to eradicate the infection while saving the limb. Through an extended anteromedial parapatellar approach a thorough debridement was performed with excision of the entire knee. The distal femur and the tibial plateau were divided back to macroscopically viable, bleeding bone. The extensor mechanism was retained. A polymethylmethacrylate cement spacer mixed with gentamycin and Imipenem was inserted to maintain limb length (Fig. 3). The micro-organism was sensitive to both antibiotics according to the initial results. Again, cultures taken in theatre isolated Proteus mirabilis.

She remained in hospital receiving IV Imipenem. During the early post-operative period her symptoms subsided and there was no evidence of wound infection.

A week later, her symptoms again worsened. She became febrile and complained of severe pain which was difficult to control even with the administration of opioids. Aspiration of 


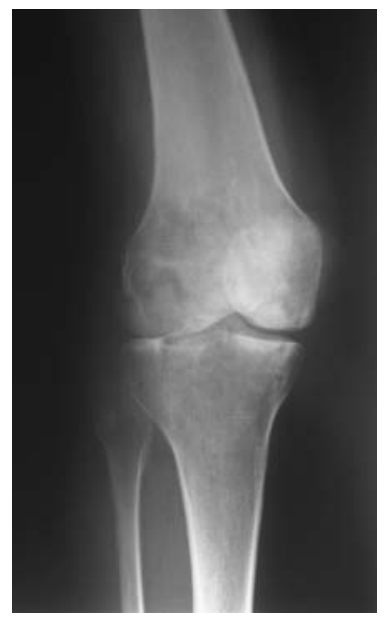

Fig. 1a

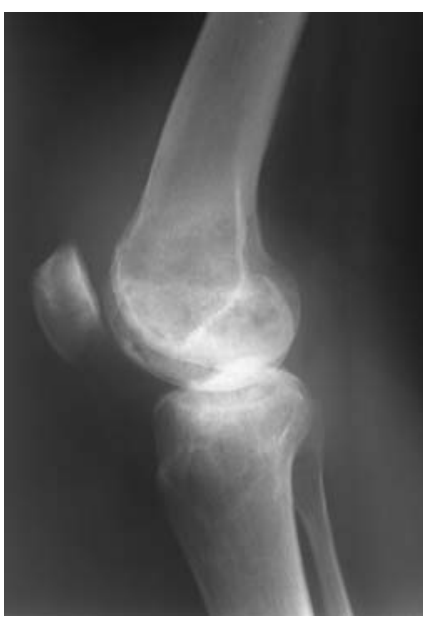

Fig. 1b

a) Plain anteroposterior and b) plain lateral radiographs of the symptomatic right knee showing destructive lytic lesions in the lower femur and upper tibia.

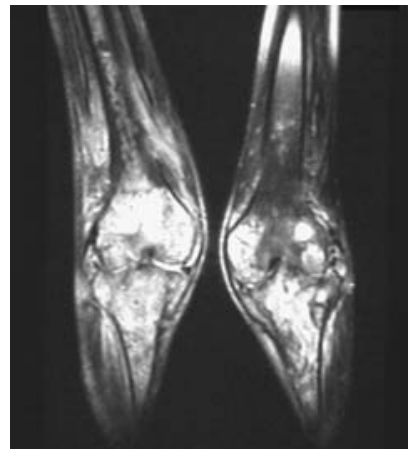

Fig. 2

Bilateral MRI showing extensive involvement of both tibiae and femora.

the affected area drained pus. In view of this it was decided to perform an above-knee amputation. At operation, pus and necrotic tissue were found at the previous site of exploration. No antibiotics were introduced into the medullary canal. The short stump was closed in layers above a Penrose drain (Sherwood Medical, Tullamore, Ireland). The cultures again confirmed Proteus mirabilis. Post-operatively, she reported considerable relief from pain. The stump healed without further complications. She was finally discharged on intramuscular (IM) Aztreonam (ER Squibb \& Sons Inc., New Brunswick, New Jersey) $1 \mathrm{~g}$, three times day for three weeks after 46 days of hospital stay, and a total of 44 days of IV Imipenem. She was able to walk using an above-knee orthosis.

The infection remained quiescent for over a year and the ESR and CRP levels returned to normal, but 14 months later she was re-admitted complaining of severe pain in her left knee. A fluctuant local swelling and marked redness were noted. The CD4 count was again above 500 cells $/ \mathrm{mm}^{3}$. She

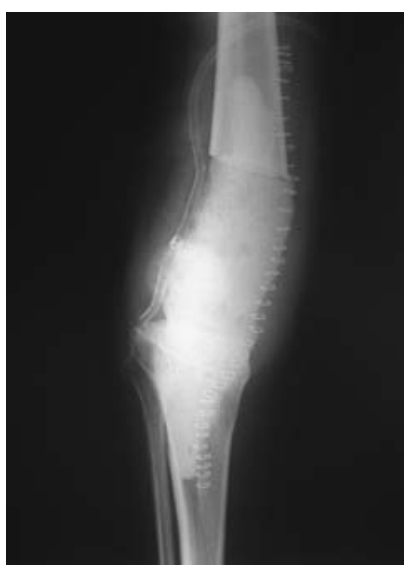

Fig. 3a

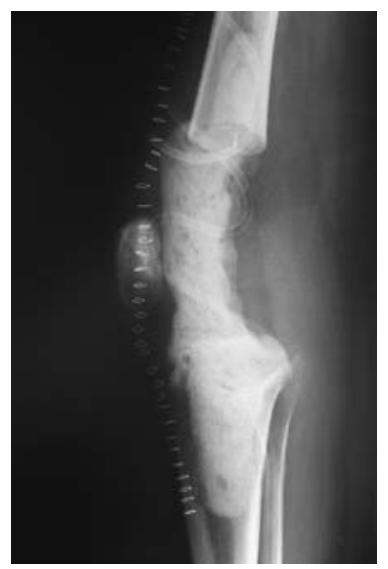

Fig. 3b
Post-operative radiographs of the right knee after excision of the joint and implantation of a cement spacer.

would not consent to an open debridement in an attempt to salvage the limb and firmly requested above-knee amputation. At operation complete destruction of the knee was evident. Again, cultures isolated Proteus mirabilis. The postoperative course was uneventful. The stump healed well and the patient has remained free from symptoms for the last two years.

\section{Discussion}

Osteomyelitis caused by infection with Proteus mirabilis is rare, especially in adults. ${ }^{1-4}$ The most common pathogen causing osteomyelitis in HIV carriers, as in the general population, is Staphylococcus aureus. ${ }^{5,6}$ The only report referring to osteomyelitis caused by Proteus mirabilis in an HIVpositive patient is by Bahebeck et al. ${ }^{7}$ In their large series of 31 musculoskeletal infections in HIV-positive patients, Proteus mirabilis was the responsible organism in six. One case involved osteomyelitis of the femur and there was one each of septic arthritis of the knee and ankle. The remaining three cases were not documented in detail. The authors reported good immediate results at three months with no recurrence at one year. We did not experience such a good clinical outcome.

The musculoskeletal manifestations of HIV infection affect up to $60 \%$ of infected patients. ${ }^{8}$ There is a wide spectrum of musculoskeletal conditions in these patients including myopathies, neoplastic conditions, inflammatory arthropathies and skeletal infections. ${ }^{6,9-11}$ However, chronic pyogenic osteomyelitis does not seem to be more common in HIV carriers than in seronegative individuals. ${ }^{5,7,11,12}$ However, it still represents one of the most serious disorders observed in HIV-positive patients, with a mortality rate of over $20 \%{ }^{5,11}$ A history of IV drug abuse, as in our patient, is a further well-known risk factor for osteomyelitis. ${ }^{6,11}$ The clinical outcome correlated with the patient's immune status, as assessed by the CD4 count in the peripheral blood. ${ }^{7,13}$ 
In our patient, the immune status at the time of infection remained satisfactory. Its evaluation was based on the CD4 count by flow cytometry ${ }^{6-7,11,14,15}$ and such patients are classified as non-, mildly, or severely immunosupressed based on a count of over 500 cells $/ \mathrm{mm}^{3}$, between 200 cells $/ \mathrm{mm}^{3}$ and 500 cells $/ \mathrm{mm}^{3}$, or less than 200 cells $/ \mathrm{mm}^{3}$ per $\mathrm{ml}$, respectively. However, our patient had discontinued her antiretroviral medication for four months before the clinical onset of the infection. The multifocal localisation of the infection suggested haematogenous spread but cultures of both blood or urine failed to isolate Proteus mirabilis.

In our patient, the infection had an indolent course for an unknown period of time. The mechanism of inoculation remained unclear. No clear explanation can be given for the multifocal localisation and the extensive bone destruction. Previous reports have also mentioned marked bony destruction in cases of Proteus mirabilis osteomyelitis of the spine and the head of the humerus, ${ }^{3,16}$ with local secretion of urease by the bacterium as a possible mechanism.

Our case indicates that in multifocal Proteus mirabilis osteomyelitis in HIV-positive patients, aggressive treatment offers the best option for a satisfactory outcome given the high mortality and morbidity of the disease.

No benefits in any form have been received or will be received from a commercial party related directly or indirectly to the subject of this article.

\section{References}

1. Kanfaoui A, Graide D, Petein M, Kengo JJ, Denis R. Proteus mirabilis osteomyelitis of the ribs in a girl with sickle cell anaemia. Eur J Pediatr 1999;158:767.
2. Kirkeby OJ, Risoe C. Proteus mirabilis osteomyelitis. $\mathrm{Br} J$ Clin Pract 1985;39:206.

3. Redfern RM, Cottam SN, Phillipson AP. Proteus infection of the spine. Spine 1988;13:439-41.

4. DeWeerd W, Kimpen JLL, Miedema CJ. Spinal osteomyelitis caused by Proteus mirabilis in a child. Eur J Pediatr 1997;156:33-4.

5. Rodriguez W. Musculoskeletal manifestations of HIV disease. AIDS Clin Care 1998; 10:49-51,56.

6. Tehranzadeh J, Ter-Oganesyan RR, Steinbach LS. Musculoskeletal disorders associated with HIV infection and AIDS. Part I: infectious musculoskeletal conditions. Skeletal Radiol 2004;33:249-59.

7. Bahebeck J, Bedimo R, Eyenga V, et al. The management of musculoskeletal infection in HIV carriers. Acta Orthop Belg 2004;70:355-60.

8. Berman A, Cahn P, Perez H, et al. Human immunodeficiency virus infection associated arthritis: clinical characteristics. J Rheumatology 1999;26:1158-62.

9. Biviji AA, Paiement GD, Steinbach LS. Musculoskeletal manifestations of human immnodeficiency virus infection. J Am Acad Orthop Surg 2002;10:312-20.

10. Plate AM, Boyle BA. Musculoskeletal manifestations of HIV infection. AIDS Read 2003;13:62,69-70,72,76.

11. Vassilopoulos D, Chalasani P, Jurado RL, Workowski K, Agudelo CA. Musculoskeletal infections in patients with human immunodeficiency virus infection. Medicine (Baltimore) 1997;76:284-94.

12. Ventura G, Gasparini G, Lucia MB, et al. Osteoarticular bacterial infections are rare in HIV-infected patients: 14 cases found among 4,023 HIV-infected patients. Acta Orthop Scand 1997;68:554-8.

13. Luck JV, Logan LR, Benson DR, Glasser DB. Human immunodeficiency virus infection: complications and outcome of orthopaedic surgery. J Am Acad Orthop Surg 1996;4:297-304

14. Casado E, Olive A, Holgado S, et al. Musculoskeletal manifestations in patients positive for human immunodeficiency virus: correlation with CD4 count. J Rheumatol 2001;28:802-4.

15. Ragni MV, Crosset LS, Herndon JH. Postoperative infection following orthopaedic surgery in human immunodeficiency virus-infected hemophiliacs with CD4 counts $<$ or $=200 / \mathrm{mm} 3$. J Arthroplasty 1995;10:716-21.

16. LaCombe MA, Funch RB. Hematogenous osteomyelitis: a report of four cases. J Maine Med Assoc 1978;69:123-6. 